\title{
Factors Associated with Medication Adherence Among Psychiatric Outpatients at Substance Abuse Risk
}

\author{
Stephen Magura ${ }^{*}, 1$, Andrew Rosenblum ${ }^{2}$ and Chunki Fong ${ }^{2}$ \\ ${ }^{I}$ The Evaluation Center, Western Michigan University, 1903 W. Michigan Ave., 4405 Ellsworth, Kalamazoo, MI 49008- \\ 5237, USA \\ ${ }^{2}$ National Development and Research Institutes, New York, NY 10010, USA
}

\begin{abstract}
Substance misuse is usually associated with poorer psychiatric medication adherence among psychiatric patients. Identifying predictors of medication adherence among patients with dual psychiatric and substance misuse problems is important because poor adherence is associated with relapse and re-hospitalization. The subjects were patients newly admitted to a psychiatric outpatient program who were prescribed psychiatric medication from different providers during the six months prior to admission; all also had substance misuse histories $(\mathrm{N}=131)$. Confidential research interviews were conducted that included a modified Medication Adherence Rating Scale (MARS) and drug toxicologies. Age (mean): 39 y; male 61\%; Black 41\%; Hispanic 38\%; White 21\%; completed high school/GED $41 \%$; DSM-IV diagnoses: major depression $26 \%$, schizoaffective $21 \%$, bipolar $16 \%$, schizophrenia $13 \%$, other $24 \%$; positive drug toxicology $55 \%$. Potentially malleable factors correlated with lower adherence were: lower friends' support for drug/alcohol abstinence, more recovery-promoting behaviors, lower satisfaction with medication, more medication side effects, lower self-efficacy for drug avoidance, and lower social support for recovery. In multivariate regression analysis, only the last three factors remained as significant predictors of adherence. Low adherence is not attributable to simply forgetting to take medication. Strengthening adherence should also include better education about side effects and the importance of adherence to sustain the benefits of medication. Psychiatrists and other medical providers should also be encouraged to address patients' adherence strategies, since the time devoted to addressing that during treatment may prevent serious adverse events such as relapse to substance abuse, treatment drop-out and re-hospitalization.
\end{abstract}

Keywords: Medication adherence, mental illness, substance abuse, comorbid disorders, psychiatric medication.

\section{INTRODUCTION}

Medication non-adherence rates are substantial among psychiatric patients. A review indicated that non-adherence ranges between $28 \%$ - $52 \%$ for major depressive disorder, $20 \%$ $50 \%$ for bipolar disorder, and $20 \%-72 \%$ for schizophrenia, while one study estimated it at $57 \%$ for anxiety disorders [1]. Substance abuse is usually associated with poorer medication adherence among psychiatric patients [2-4]. Co-occurring psychiatric and substance use disorders are common; in the U.S. 8.9 million people have both past year mental illness and dependence on or abuse of illicit drugs or alcohol [5]. Recognizing predictors of medication adherence among patients with dual psychiatric and substance use disorders is important because poor medication adherence is associated with relapse to substance abuse, re-hospitalization, homelessness and lower quality of life [1,6]. Although there have been many studies of psychiatric medication adherence, there are few such studies focusing specifically on patients with co-occurring psychiatric and substance abuse disorders.

The objectives of the present study are:

To determine the extent of psychiatric medication non-adherence among a sample of psychiatric outpatients with substance abuse histories.

*Address correspondence to this author at the Evaluation Center, Western Michigan University, 1903 W. Michigan Ave., 4405 Ellsworth, Kalamazoo, MI 49008-5237, USA; Tel: 269-387-5895; Fax: 269-387-5923;

E-mail: stephen.magura@wmich.edu
(2) To determine the malleable factors associated with non-adherence which could suggest means for improving adherence in this population.

By malleable factors we mean behaviors, attitudes, beliefs and social environment elements that could be targets for intervention, rather than fixed factors such as sociodemographics (e.g., age, gender, education) and background characteristics (e.g., criminal history, treatment history, past alcohol/drug use). The present study includes measures of three types of malleable risk factors as specified in Julius et al., [1] theory of psychiatric medication adherence: medication-related risk factors, psychological risk factors, and social/environmental risk factors,

The specific hypotheses involving malleable risk factors and their justification are:

1. More or more severe medication side effects will be related to lower psychiatric medication adherence. Numerous previous studies have reported this relationship for psychiatric patients [7-11] including among mentally ill substance abusers $[6,12]$.

2. Higher psychological risk factors will be related to lower psychiatric medication adherence. Psychological risk factors which have been shown to be related to lower medication adherence are: poor insight into psychiatric illness and lack of readiness for treatment $[3,13,14]$ negative attitudes toward 
medication $[2,3,15]$, and inadequate self-efficacy regarding symptoms $[6,14,16]$.

3. More or more severe social and environment risk factors will be related to lower psychiatric medication adherence. Social/environmental risk factors which have been shown to be related to lower medication adherence are: Lack of social support [1719], lack of supportive services [20]; social instability [21], no or lower attendance at peer recovery groups for mentally ill or dually-diagnosed individuals [6, 22], no spouse who can serve as a caregiver [23] and independent living arrangement [11].

\section{METHODS}

\section{Setting}

The setting was a psychiatric continuing day treatment program located in the Bronx, New York City. Patients are referred to this program from various mental health and addiction treatment settings, including psychiatric inpatient units, mental health residences, other outpatient mental health clinics and outpatient addiction treatment clinics; or were self-referred through community contacts. This program provides services both for persons with single psychiatric disorders and those dually diagnosed with psychiatric and substance use disorders.

\section{Study Design}

The present study is an uncontrolled cross-sectional study of patients newly admitted to this outpatient program from March 2003 to December 2005. The data were collected by researchers primarily through confidential personal interviews with the patients usually within a week after admission. The questions pertinent to this study referred to attitudes and beliefs at the time of admission and behaviors/situations occurring in the month before admission. The study also compiled psychiatric diagnoses assigned by program psychiatrists at the time of admission and toxicologies for drugs of abuse obtained on biological specimens collected by the researchers at the time of the interview.

\section{Study Sample and Procedures}

Patients admitted to the program were referred by a program intake counselor to a study research assistant for eligibility assessment. Patients were excluded from the study if they were younger than age 18, did not understand or speak English, appeared intoxicated on drugs or alcohol, carried a diagnosis of mental retardation, were deemed actively psychotic by the program's intake coordinator, or appeared unable to understand and give informed consent. The present study includes only those newly admitted patients who were prescribed psychiatric medication from different providers during the six months prior to admission and were administered a measure of medication adherence by the study $(\mathrm{N}=131)$. All patients who agreed to participate in the study signed an informed consent. Participants received compensation of $\$ 20.00$ for an in-person research interview and biological specimens for drug testing. The study protocol was approved by the Institutional Review Boards of the host research site and the organization that conducted the study.

\section{Measures}

Subject Background Variables. The study included sociodemographic, psychiatric and alcohol/drug use background variables at study intake. The subject's sociodemographic characteristics were obtained from the baseline interview: age, gender, race/ethnicity, education, public assistance and criminality. DSM-IV psychiatric disorder diagnoses were made by the program psychiatrists at admission and abstracted by the researchers from the medical records. An index of alcohol/drug use intensity in the 90 days before admission was constructed as follows. The subjects were asked on how many days in the last 90 days that they used each of a list of substances: alcohol (beer, wine and/or liquor), marijuana, cocaine/crack, heroin, and other opioids, "street" methadone, hallucinogens, barbiturates, sedatives, other stimulants, Ecstasy and inhalants. Each substance was described by common brand or street names. The numbers of days were added to create a heuristic index of alcohol/drug use; the higher the score, the more alcohol and drug use. In addition, urine and head hair specimens were obtained by the researchers at admission for drug toxicology. Urine toxicology was conducted by on-site immunoassay (Roche Test Cup) for opiates (morphine), cocaine metabolite (benzoylecgonine), marijuana (THC), phencyclidine, and amphetamines. Urine specimens were obtained for $96.8 \%$ of the sample. Hair analysis was conducted by radioimmunoassay by Psychemedics Corporation for opiates and cocaine metabolite. A 1.5" specimen of head hair as measured from the scalp was analyzed, which gives an approximate window of detection of 90 days; hair specimens were obtained for $55.4 \%$ of the sample. The primary reason for not obtaining hair was men's predominant short hair styles ("buzz cuts") that either made it impossible to cut hair or would have left an obvious bald spot not acceptable to respondents. If any of these tests was positive, the subject was classified as drug toxicologypositive. Alcohol/drug use history was defined as any mention on the personal interview of alcohol or (nonmedical) drug use or treatment in the subject's lifetime.

Psychiatric Medication Adherence. The Medication Adherence Rating Scale (MARS) [24] was modified by dropping Factor 3, consisting of two items ("medication makes me feel like zombie; feel tired and sluggish"), to avoid overlap with the medication side-effects measure below. The eight items are listed in Table 3; modified MARS-8 $v$ MARS: $\mathrm{r}=0.94$.

Medication-related Risk Factor for Non-adherence. Medication side effects were measured by presenting subjects with a list of 16 items (e.g., sleep problems; shaking/tremors; restlessness/jitteriness) with responses that ranged from "not at all" to "very much".

Psychological Risk Factors for Non-adherence. Four such factors were measured; these were:

Self-Efficacy for Mental Health Recovery (Mental Health Confidence Scale) [25]. 16 items (e.g., how confident are you right now that you can: Deal with symptoms related to your mental illness? Face a bad day?).

Self-Efficacy for Drug Avoidance (DASES) [26]. Seven items (e.g., imagine you are home alone, it's a dull weekend 
and you are bored. Would you give in the urge to get stoned/loaded?)

Readiness to Change Questionnaire (RCQ) [27]. This is a 12 -item scale which is based on Prochaska \& Di Clemente's Trans-theoretical Model. Items for the RCQ are reworded to represent both drinking and drug use (e.g., "sometimes I think I should cut down on my drug use [or my drinking])".

Satisfaction with Medication. One question asked: "How satisfied were you with the psychiatric medications you were taking before you came to this program?" The responses range from "not at all satisfied" to "very satisfied."

Social/Environmental Risk Factors for Non-Adherence. Three such factors were measured:

Recovery Support. Six items adapted from the Social Support for Recovery scale [28]. E.g., in the past month, how much support were your friends giving you in recovering: from mental illness? From drug/alcohol abuse?

Friends' Support for Abstinence. This is an 8-item index adapted from the Social Network Influence Scale [29]. E.g., "my friends offer advice about quitting drugs or alcohol without nagging."

Recovery-Promoting Behaviors. (Processes of Change Questionnaire) [30]. 10 items (e.g., I remove things from my home/work that remind me of drinking/using drugs).

Psychiatric Symptoms. (Colorado Symptoms Index -CSI) [31]. Higher adherence is expected to be correlated with less psychiatric symptomology, although the direction of effect is not being hypothesized. The CSI was developed specifically for assessment of symptoms at levels experienced by people diagnosed with mental illness. 13 items (e.g., "how often did you: feel nervous or tense? Feel depressed?)

\section{Data Analysis}

Analyses include descriptive statistics, bivariate correlations and stepwise multivariate linear regression. In the latter, medication adherence (MARS-8) was the dependent variable and the background characteristics and risk factors for non-adherence were the independent variables. The level for statistical significance of independent variables was set at $\mathrm{p}<.05$ (2-tailed). The PASW Statistics 18 software was used for all statistical analyses.

\section{RESULTS}

\section{Characteristics of the Sample}

The majority of the sample was male and from racial/ethnic minority groups, with an average age of 39 years. Less than half had graduated from high school and most had arrest histories and were supported by public assistance or disability. All reported substance misuse histories and 55\% were drug-positive by toxicology at program admission (Table 1). The most frequent primary psychiatric diagnosis was major depression, followed by schizoaffective, bipolar, schizophrenic and other mood disorders (Table 2). None of the variables in Tables $\mathbf{1}$ or $\mathbf{2}$ had significant bivariate correlations with MARS-8 (medication adherence).
Table 1. Sample Background Variables $(\mathrm{N}=131)$

\begin{tabular}{|lcc|}
\hline & & (N) \\
\hline \hline Age in Years (mean, sd) & $39.2,10.7$ & $(131)$ \\
Male & $61 \%$ & $(80)$ \\
Black & $41 \%$ & $(54)$ \\
Hispanic & $38 \%$ & $(50)$ \\
White & $21 \%$ & $(27)$ \\
Completed High School/GED & $41 \%$ & $(54)$ \\
Public Assistance/Disability & $73 \%$ & $(95)$ \\
Arrested as Adult & $71 \%$ & $(93)$ \\
Alcohol/Drug Use Intensity (mean, sd) & $6.0,40.5$ & $(131)$ \\
Drug Toxicology Positive & $55 \%$ & $(131)$ \\
Alcohol/Drug Use History & $100 \%$ & $(131)$ \\
\hline
\end{tabular}

Table 2. DSM-IV Axis I Psychiatric Diagnoses

\begin{tabular}{|lcc|}
\hline & Percent & (N) \\
\hline \hline Major Depression & 26 & $(34)$ \\
Schizoaffective & 21 & $(28)$ \\
Bipolar & 16 & $(21)$ \\
Schizophrenia & 13 & $(17)$ \\
Other Mood & 9 & $(12)$ \\
Psychotic-NOS & 7 & $(9)$ \\
Anxiety & 3 & $(4)$ \\
Other & 5 & $(6)$ \\
\hline
\end{tabular}

A mean of 2.6 non-adherence items were endorsed on the 8-item modified MARS (Table 3). The most frequently endorsed items were: unnatural to be controlled by medication (47\%); careless at times (44\%) and sometimes stopped when felt better (44\%). Forgetting to take medication was one of the less frequently endorsed items (31\%). The MARS was correlated significantly with the Colorado Symptoms Index (CSI) $(r=0.22, p<.05)$. However, the direction of effect is unclear. Patients with more severe symptoms tend to have more complex medication regimens and complexity has been found to predict medication nonadherence [32, 33]. But also, we might expect lower medication adherence that occurs for other reasons to result in poorer psychiatric symptom control. Because the direction of effect is uncertain, the CSI was not included in the subsequent analysis of risk factors for low medication adherence.

The initial prediction analysis correlated each risk variable with the modified MARS; note that higher MARS scores indicates lower adherence (Table 4). Six risk variables were significantly correlated with the modified MARS, all in the hypothesized directions. One was the medication related risk factor (i.e., medication side effects), two were 
Table 3. Medication Adherence Rating Scale (MARS-8)

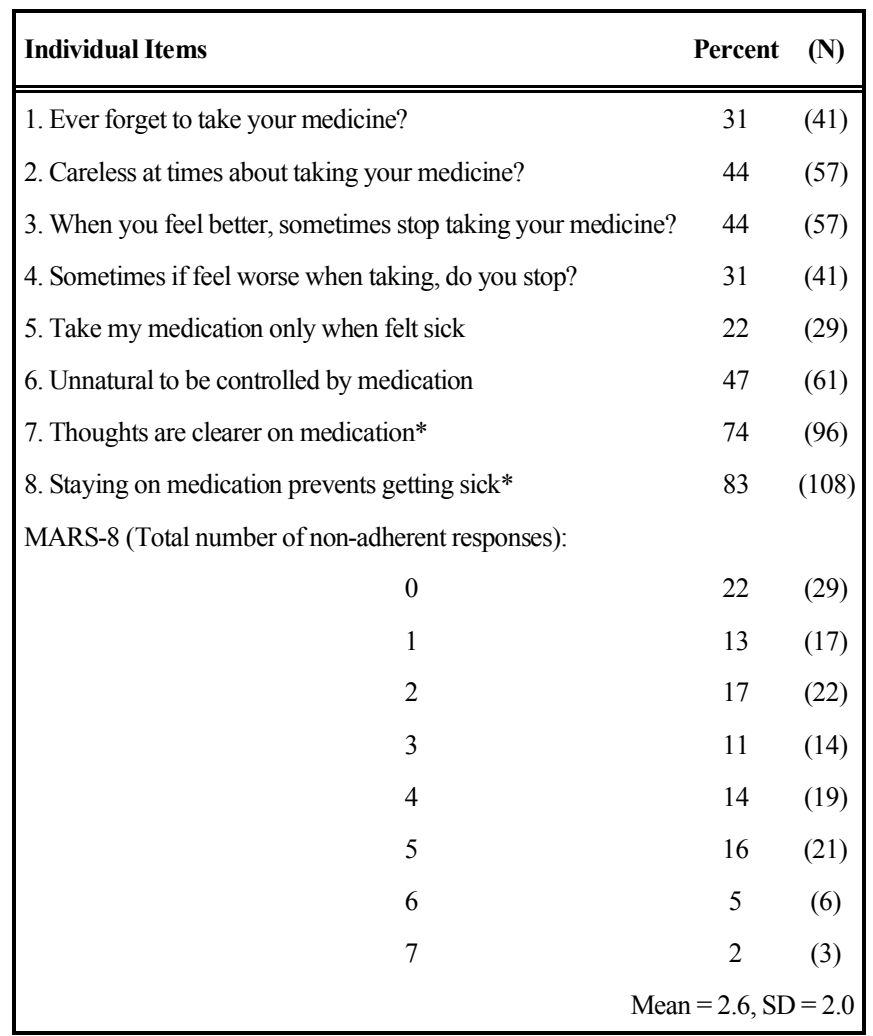

*Reverse scoring for MARS-8 total; disagreeing with this item connotes nonadherence.

Higher total additive score $=$ less medication adherence.

psychological risk factors (self-efficacy for drug avoidance; satisfaction with medication), and three were social/environmental risk factors (recovery support; friends' support for abstinence, recovery-promoting behaviors).

Because the risk variables were also inter-correlated, a stepwise multivariate linear regression analysis was conducted to determine the most parsimonious set of predictors of medication adherence. An additional important question is whether these malleable risk factors explain variance in adherence beyond the variance that can be explained by patient background characteristics. Thus, the background characteristics as shown in Table 1 were entered as a block in first step of the regression, explaining $8 \%$ of the variance in adherence, However, none of these variables, including alcohol/drug use intensity, were significant individually (Table 5). The second step entered the six hypothesized risk factors that were significant in the bivariate analysis as shown in Table 4, resulting in an increase to $23 \%$ in variance explained in adherence. The third step consisted of backward elimination of the hypothesized risk factors. This involved starting with all risk factor variables entered in step 2, testing them individually for statistical significance, and deleting any that are not significant, until only statistically significant risk factors remain in the model. (The background characteristics entered in step 1 continued to be retained as control variables in the analysis.) The results of the backward elimination were the retention of three risk factors - self-efficacy for drug avoidance, medication side effects and recovery support.

The final model with these three risk factor explained $21 \%$ of the variance in adherence, which was an improvement of $21 \%-8 \%=13 \%$ over the background variables alone in explained variance.

\section{DISCUSSION}

In multivariate analysis, lower medication adherence was associated with a medication-related variable (more side effects), a cognitive variable (lower self-efficacy for drug avoidance) and a social factor (lower support for recovery). This wide variety of predictors suggests that simply providing technical aids to aid memory (e.g., pill dispensers with alarms), while potentially useful, may be insufficient to increase adherence. This is also supported by the fact that relatively few patients attributed low adherence to forgetting to take medication.

With regard to side effects, one-third of the patients also said their psychiatrists did not spend enough time with them to explain side effects or were "rushed." Strengthening adherence should include better education about side effects and the importance of adherence to sustain the benefits of medication. This education, which might also involve assertiveness training, should encourage patients to discuss their symptoms and side effects with their psychiatrists,

Table 4. Significant Predictors of Medication (non) Adherence (MARS-8)

\begin{tabular}{|lccccc|}
\hline & Mean & SD & (N) & Bivariate Correlation with MARS-8 & P-Value \\
\hline \hline Medication-Related Risk Factor & & & & & .31 \\
Medication Side Effects & 5.4 & 4.7 & $(131)$ & & .000 \\
Psychological Risk Factors & & & & -.24 \\
Self-Efficacy for Drug Avoidance & 5.0 & 1.6 & $(127)$ & -.19 & .006 \\
Satisfaction with Medication & 2.2 & 0.9 & $(131)$ & .033 \\
Social/Environmental Risk Factors & & & & -.23 \\
Recovery Support & 2.4 & 1.0 & $(131)$ & -.20 & .010 \\
Friend's Support for Abstinence & 3.3 & 0.7 & $(131)$ & .21 \\
Recovery-Promoting Behaviors & 2.3 & 0.9 & $(125)$ & .018 \\
\hline
\end{tabular}


Table 5. Stepwise Multivariate Linear Regression of Predictors on MARS-8

\begin{tabular}{|c|c|c|c|}
\hline & Unstandardized Beta & Standard Error & P-Value \\
\hline \multicolumn{4}{|l|}{ Step 1: Enter all Background Variables } \\
\hline Age & -.001 & .002 & .545 \\
\hline Male & -.001 & .048 & .984 \\
\hline Black & .010 & .060 & .872 \\
\hline Hispanic & -.018 & .058 & .759 \\
\hline Completed High School/GED & .016 & .047 & .731 \\
\hline Public Assistance/Disability & .068 & .052 & .191 \\
\hline Arrested as Adult & .096 & .053 & .071 \\
\hline Alcohol/Drug Use Intensity & .001 & .001 & .107 \\
\hline Drug Toxicology Positive & -.066 & .050 & .193 \\
\hline \multicolumn{4}{|l|}{$\mathrm{R}^{2}=.08$} \\
\hline \multicolumn{4}{|c|}{ Step 2: Add Significant Hypothesized Risk Factors* } \\
\hline Age & -.002 & .002 & .304 \\
\hline Male & -.017 & .048 & .718 \\
\hline Black & .026 & .057 & .647 \\
\hline Hispanic & -.021 & .055 & .702 \\
\hline Completed High School & .010 & .045 & .820 \\
\hline Public Assistance/Disability & .073 & .049 & .141 \\
\hline Arrested as Adult & .044 & .052 & .398 \\
\hline Alcohol/Drug Use Intensity & .000 & .001 & .785 \\
\hline Drug Toxicology Positive & -.044 & .049 & .369 \\
\hline Medication Side Effects & .014 & .005 & .006 \\
\hline Self-Efficacy for Drug Avoidance & -.022 & .016 & .173 \\
\hline Satisfaction with Medication & .020 & .026 & .436 \\
\hline Recovery Support & -.032 & .026 & .224 \\
\hline Friend's Support for Abstinence & -.015 & .036 & .681 \\
\hline Recovery-Promoting Behaviors & -.029 & .029 & .320 \\
\hline \multicolumn{4}{|l|}{$\mathrm{R}^{2}=.23$} \\
\hline \multicolumn{4}{|c|}{ Step 3: Backward Elimination of Hypothesized Risk Factors } \\
\hline Age & -.002 & .002 & .286 \\
\hline Male & -.009 & .047 & .846 \\
\hline Black & .032 & .057 & .575 \\
\hline Hispanic & -.017 & .055 & .758 \\
\hline Completed High School & .005 & .044 & .910 \\
\hline Public Assistance/Disability & .076 & .049 & .124 \\
\hline Arrested as Adult & .050 & .051 & .324 \\
\hline Alcohol/Drug Use Intensity & .000 & .001 & .579 \\
\hline Drug Toxicology Positive & -.054 & .048 & .263 \\
\hline Medication Side Effects & .013 & .005 & .008 \\
\hline Self-Efficacy for Drug Avoidance & -.029 & .014 & .043 \\
\hline Recovery Support & -.046 & .023 & .046 \\
\hline $\mathrm{R}^{2}=.21$ & & & \\
\hline
\end{tabular}


rather than simply to stop taking medication. Psychiatrists should also be encouraged to address patients' adherence strategies, since the time devoted to addressing that during treatment may prevent serious adverse events such as relapse, treatment drop-out and hospitalization. An alternative cost-effective method could use other medical providers such as nurse practitioners or physician assistants to educate patients about medication adherence.

Patients with greater adherence problems also reported lower support for recovery and lower self-efficacy for drug avoidance. Several studies have shown that "dual focus" self-help groups providing social support for recovery for individuals with psychiatric and substance use disorders can increase medication adherence $[6,34,35]$. Such self-help groups also assist members in avoiding situations where they may be exposed to illicit drug users who are not likely to support medication adherence. Although there was no relation between Alcoholics Anonymous participation and medication adherence, but it must be noted that psychiatric medication is not considered a suitable topic of discussion at AA meetings [36, 37]. Participation in "dual focus" self-help groups should be encouraged by mental health and addiction treatment clinicians who work with patients with cooccurring disorders.

Lower self-efficacy for drug avoidance may also be associated with self-medication behaviors, which are not unusual in this type of population. Specifically, low selfefficacy for drug avoidance may be a proxy for selfmedication of psychiatric symptoms with alcohol or with drugs of abuse, and the more such self-medication, the lower would be adherence to prescribed medications.

It might be surprising that the intensity of substance use was not associated with medication adherence, but this is not necessarily inconsistent with prior research, which generally has found differences in adherence between those with and without substance use, rather than between those varying in degrees of use [3]. In our study virtually all subjects had both lifetime and recent histories of use. It may be that the degree of use does not impair the ability of these patients to take their medication, perhaps because they have adapted to functioning with some substances of abuse "on board."

\section{Study Limitations}

The study is based on a cross-sectional analysis that requires caution in making causal inferences about predictors of adherence. The study's sample size does not allow determination of whether the results apply to all individual psychiatric diagnoses or different categories of substance users such as alcoholics or drug users. The study was conducted with patients in one program and thus generalizability to all patients with both mental health and substance misuse problems may be limited.

\section{Directions for Future Research}

It would be useful to test adherence interventions that combine peer support, such as dual focus self-help groups, with increased patient-centered education efforts by medical staff. There should also be continuing research on improved measures of medication adherence. For instance, the modified version of the MARS used in this study may be an improved option.

\section{ACKNOWLEDGEMENT}

Funded by National Institute on Drug Abuse, Grant \# R01 DA015912.

\section{REFERENCES}

[1] Julius RJ, Novitsky MA Jr, Dubin, WR. Medication adherence: a review of the literature and implications for clinical practice. J Psychiatr Pract 2009; 15: 34-44.

[2] Sowers W, Golden S. Psychotropic medication management in persons with co-occurring psychiatric and substance abuse disorders. J Psychoactive Drugs 1999; 3: 59-70.

[3] Lacro JP, Dunn LB, Dolder CR, et al. Prevalence of and risk factors for medication nonadherence in patients with schizophrenia: a comprehensive review of recent literature. J Clin Psychiatry 2002; 63: 892-909.

[4] Weiss RD. Adherence to pharmacotherapy in patients with alcohol and opioid dependence. Addiction 2004; 99(11): 1382-92.

[5] http://www.oas.samhsa.gov/NSDUH/2k9NSDUH/MH/tabs/SectlpeMHta bs.htm\#Tab1.10B [Retrieved March 18, 2011].

[6] Magura S, Laudet A, Mahmood D, et al. Medication adherence and participation in self-help groups designed for dually-diagnosed persons. Psychiatric Serv 2002; 53: 310-6.

[7] Hoge SK, Appelbaum PS, Lawlor T, et al. A prospective, multicenter study of patients' refusal of antipsychotic medication. Arch Gen Psychiatry 1990; 47(10): 949-56.

[8] Robinson D, Woerner MG, Alvir JM, et al. Predictors of relapse following response from a first episode of schizophrenia or schizoaffective disorder. Arch Gen Psychiatry 1999; 56(3): 241-7.

[9] Perkins DO, Hongbin G, Weiden PJ, et al. Predictors of treatment discontinuation and medication adherence in patients recovering from a first episode of schizophrenia, schizophreniform disorder or schizoaffective disorder: a randomized, double-blind, flexible dose, multicenter study. J Clin Psychiatry 2008; 69: 106-13.

[10] Ruscher SM, de Wit R, Mazmanian D. Psychiatric patients' attitudes about medication and factors affecting noncompliance. Psychiatric Serv 1997; 48(1): 82-5.

[11] Sajotovic M, Valenstein M, Blow F, et al. Treatment adherence with lithium and anticonvulsive medications among patients with bipolar disorder. Psychiatric Serv 2007; 58: 855-63.

[12] Weiss RD, Greenfield SF, Najavits LM, et al. Medication compliance among patients with bipolar disorder and substance use disorder. J Clin Psychiatry 1998; 59(4): 172-4.

[13] Pyne JM, Bean D, Sullivan G. Characteristics of patients with schizophrenia who do not believe they are mentally ill. J Nerv Ment Dis 2001; 189(3): 146-53.

[14] Velligan DI, Weiden PJ, Sajatovic M, et al. Expert consensus panel on adherence problems in serious and persistent mental illness. The expert consensus guideline series: adherence problems in patients with serious and persistent mental illness. J Clin Psychiatry 2009; 70(Supp14): 1-46.

[15] Day JC, Bentall RP, Roberts C, et al. Attitudes toward antipsychotic medication. Arch Gen Psychiatry 2005; 62: 717-24.

[16] Naar-King S, Templin T, Wright K, Frey M, Parsons JT, Lam P. Psychosocial factors and medication adherence in HIV-positive youth. AIDS Patient Care STDS 2006; 20(1): 44-7.

[17] Anthony KH. Helping partnerships that facilitate recovery from severe mental illness. J Psychosoc Nurs Ment Health Serv 2008; 46(7): 24-8, 29-33.

[18] Hudson TJ, Owen RR, Thrush CR, et al. A pilot study of barriers to medication adherence in schizophrenia. J Clin Psychiatry 2004; 65(2): 211-6.

[19] Kikkert MJ, Schene AH, Koeter MW, et al. Medication adherence in schizophrenia: exploring patients', carers' and professionals' views. Schizophrenia Bulletin 2006; 32(4): 786-94.

[20] Zygmunt A. Olfson M. Boyer CA, Mechanic D. Interventions to improve medication adherence in schizophrenia. Am J Psychiatry 2002; 159(10): 1653-64.

[21] Elbogen EB, Swanson JW, Swartz MS, Van Dorn R. Medication non adherence and substance abuse in psychotic disorders: impact of depressive symptoms and social stability. J Nerv Ment Disease 2005; 193(10): 673-9.

[22] Kurtz LS. Use of lithium and other medications by members of a self-help group. Lithium 1990; 1: 125-6. 
[23] Sher I, McGinn L, Sirey JA, Meyers B. Effects of caregivers' perceived stigma and causal beliefs on patients' adherence to antidepressant treatment. Psychiatric Serv 2005; 56(5): 564-9

[24] Thompson, K, Kulkarni J, Sergejew, AA. Reliability and validity of a new Medication Adherence Rating Scale (MARS) for the psychoses. Schizophr Res 2000; 42; 241-7.

[25] Carpinello SE, Knight EL, Markowitz FE, et al. The development of the mental health confidence scale: a measure of self-efficacy in individuals diagnosed with mental disorders. Psychiatr Rehabil J 2000; 23: 236-43.

[26] Martin GW, Wilkinson DA, Poulos CX. The Drug Avoidance SelfEfficacy Scale. J Subst Abuse 1995; 7: 151-63.

[27] Rollnick S, Heather N, Gold R, et al. Development of a short "readiness to change" questionnaire for use in brief opportunistic interventions among excessive drinkers. Brit J Addic 1992: 87: 743-54.

[28] Laudet A, Magura S, Vogel H, Knight E. Support, mutual aid and recovery from dual diagnosis. Community Ment Health 2000; 36:457-76.

[29] Humphreys K, Huebsch PD, Finney JW, Moos RH. A comparative evaluation of substance abuse treatment: V. Substance abuse treatment can enhance the effectiveness of self-help groups. Alcoholism: Clin Exp Res1999; 23: 558-63.
[30] Prochaska JO, Velicer WF, DiClemente CC, et al. Measuring processes of change: applications to the cessation of smoking. J Consult Clin Psychol1988; 56: 520-8.

[31] Shern DL, Wilson NZ, Coen AS, et al. Client outcomes II: longitudinal client data from the Colorado Treatment Outcome Study. The Milbank Quarterly 1994: 72: 123-48.

[32] Cramer JA, Rosenheck R. Compliance with medication regimens for mental and physical disorders. Psychiatr Serv 1998; 49(2): 196201.

[33] Claxton AJ, Cramer J, Pierce CA. Systematic review of the associations between dose regimens and medication compliance. Clin Ther 2001; 23(8): 1296-310.

[34] Magura S, Rosenblum A, Villano C, et al. Mutual aid for cooccurring disorders: a quasi-experimental outcome study. Am J Drug Alcohol Abuse 2008; 34: 61-74.

[35] Magura S, Villano CL, Rosenblum A, et al. Consumer evaluation of dual focus mutual aid. J Dual Diagn 2008; 4: 170-85.

[36] Noordsy D, Schwab B, Fox L, Drake R. The role of self-help programs in the rehabilitation process of persons with severe mental illness and substance use disorders. Community Ment Health 1996; 32: 71-81.

[37] Vogel HS, Knight EL, Laudet AB, et al. Double trouble in recovery: self-help for the dually-diagnosed. Psychiatric Rehab J 1998; 21: 356-64.

(C) Magura et al.; Licensee Bentham Open.

This is an open access article licensed under the terms of the Creative Commons Attribution Non-Commercial License (http://creativecommons.org/licenses/ by-nc/3.0/) which permits unrestricted, non-commercial use, distribution and reproduction in any medium, provided the work is properly cited. 